\title{
ELEMENTOS DEL ESTUDIO DE LA DEMANDA SOCIAL Y DEL MERCADO OCUPACIONAL DE LA CARRERA PROFESIONAL DE CONTABILIDAD
}

\author{
ELEMENTS OF THE STUDY OF SOCIAL AND MARKET DEMAND \\ OCCUPATIONAL CAREER ACCOUNTING FOR NATIONAL \\ ACCREDITATION PURPOSES
}

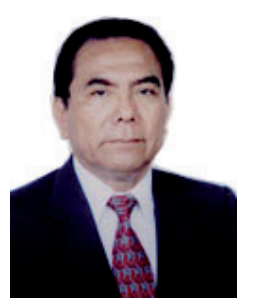

Adrián Alejandro Flores Konja

Docente Principal de la Facultad de Ciencias Contables

Manuel Alberto Hidalgo Tupia

Docente Auxiliar de la Facultad de Ciencias Contables

Universidad Nacional Mayor de San Marcos-UNMSM / Lima-Perú

[Recepción: Julio de 2013/ Conformidad: Agosto de 2013]

\section{RESUMEN}

El artículo presenta los resultados y las conclusiones parciales de la investigación titulada: "Elementos del estudio de la demanda social y del mercado ocupacional de la carrera profesional de contabilidad para los fines de la acreditación nacional”, en ejecución en el presente periodo 2013.

El estudio ha sido requerido expresamente por el Modelo de Calidad de CONEAU. Sin embargo, su importancia trasciende el aspecto formal del requerimiento para la acreditación, siendo importantísimo para el proceso de mejora continua en la Facultad de Ciencias Contables.

El Ministerio de Educación señala un problema de pertinencia en la Universidad peruana, pues se presentan por un lado, carreras que no tienen postulantes y por el otro, carreras con tantos egresados, que muchos de ellos pueden no ser absorbidos por el mercado ocupacional propio de la carrera, este estudio permite enfocar ambas situaciones.

En el caso de la Carrera Profesional de Contabilidad se parte de una valoración positiva, tanto de la demanda social de la carrera profesional ofrecida por nuestra Facultad de Ciencias Contables como de la absorción pronta de nuestros egresados por el mercado ocupacional. La formulación del estudio nos permitirá corroborar, mantener y mejorar la ventaja alcanzada por nuestra Facultad.

Palabras Claves: Carrera profesional, mercado ocupacional, demanda social, calidad educativa, acreditación, CONEAU.

\section{ABSTRACT}

The article presents the partial results and conclusions of the research entitled: "Elements of the study of social and market demand occupational career accounting for national accreditation purposes", running in this period 2013.

The study has been expressly required by the CONEAU Quality Model. However, its importance transcends the formal aspect of the requirement for accreditation, being important for the process of continuous improvement in the School of Accounting. The Ministry of Education reports a problem of relevance in the Peruvian University, as presented by one side do not have careers that applicants and on the other races with many graduates, many of them can not be absorbed by the job market 's own the race, this study allows focusing both.

For the Accounting Career is part of a positive assessment of both social demand careers offered by our School of Accounting and the rapid absorption of our graduates for the job market. The study design will allow us to corroborate, maintain and improve the advantage achieved by our faculty.

Keywords: Career, job market, social demand, quality of education, accreditation, CONEAU.

\footnotetext{
* Doctor en Ciencias Contables. Magister en Contabilidad con Mención en Auditoría. Contador Público Colegiado. UNMSM.

Email: afloreskonja@yahoo.es

**Magister en Educación. Economista. Email: mhidalgot@unmsm.edu.pe
} 


\section{INTRODUCCIÓN}

Según la fuente mas autorizada respecto al mercado ocupacional de las carreras universitarias en el Perú, es la investigación de Luis Piscoya Hermoza, "Formación Universitaria vs. Mercado Laboral", publicada por la Asamblea Nacional de Rectores. Señala que de las 188 carreras ofrecidas por el Sistema Universitario Peruano, algunas de ellas no tienen demanda. Las empresas encuestadas en el estudio mencionado "demandan sólo 86 " de las 188 ; pero de otra parte, de ese mismo grupo de carreras en el año 2006, "se registran 83 carreras sin postulantes". Es decir, carreras que no gozan de la aceptación ni de los empleadores ni de los educandos; dicho en los términos escogidos por CONEAU - Consejo de Evaluación, Acreditación y Certificación de la Calidad de la Educación Superior Universitaria, no tienen mercado ocupacional ni demanda social.

Piscoya señala que Contabilidad es la carrera con mayor demanda en la actualidad, superando a las demás, pero que las empresas y organizaciones encuestadas afirman que la Contabilidad es la tercera carrera "de futuro", superada por Administración e Ingeniería Industrial.

En la investigación en progreso, se dilucidará los factores que deben considerarse en el Estudio de la Demanda Social y del Mercado Ocupacional y que deberá realizar la Facultad para un mejor conocimiento y toma de decisiones. Se formularán el contenido del estudio; así como, los instrumentos de recolección de información para el Estudio.

La Facultad de Ciencias Contables, que cuenta con la Acreditación Internacional de la Carrera Pro- fesional de Contabilidad, su principal carrera yla más antigua otorgada por la acreditadora internacional CACSLA - CACECA, ha iniciado el proceso de la acreditación nacional bajo el modelo de CONEAU, por lo cual el tema es pertinente.

La importancia de la investigación radica también, en que se aprovechará para preguntar sobre las competencias profesionales específicas requeridas por los empleadores, que pueden reforzar el planeamiento curricular de la carrera.

Por ser una exigencia reciente del CONEAU, no existen estudios de demanda social y demanda ocupacional de las carreras profesionales, no se ha planteado una metodología para prepararlos, por lo que constituyen un conocimiento por adquirir.

Los objetivos de la investigación son: Conocer cuáles son los elementos del estudio de la demanda social y demanda ocupacional de la carrera profesional de Contabilidad para un direccionamiento más eficiente de la planificación curricular.

La meta específica es determinar la coherencia entre los elementos del estudio de la demanda social y demanda ocupacional de la carrera profesional de Contabilidad para los fines de la acreditación nacional con la planificación estratégica y curricular de la carrera profesional.

\section{MARCO CONCEPTUAL}

En el presente estudio, concebimos dos mercados interrelacionados. En primer lugar, el mercado de formación profesional en orden cronológico y el mercado ocupacional profesional, en segundo lugar, cuyas relaciones se significan en el gráfico siguiente: 


\section{Gráfico $\mathrm{N}^{\circ} 1$ \\ DEMANDA SOCIAL Y MERCADO OCUPACIONAL DE UNA CARRERA PROFESIONAL}

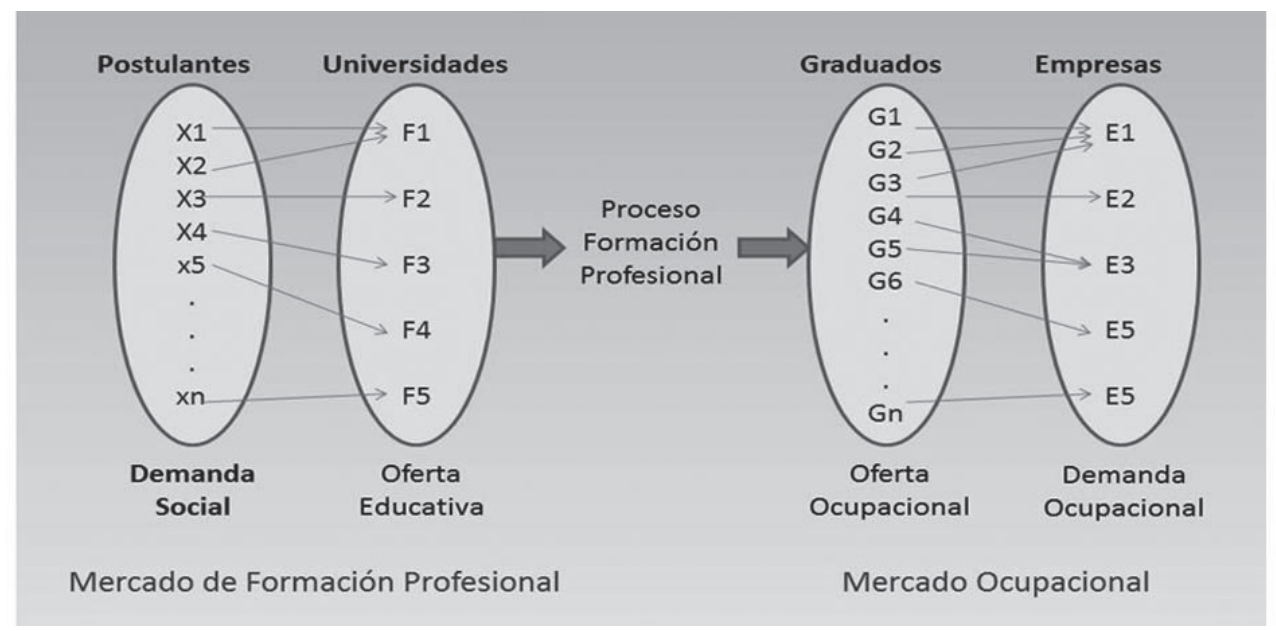

Haciendo una analogía con los estudios de mercado para productos o servicios, podernos aportar las siguientes definiciones propias:

\section{La demanda social de una carrera profesional} es "el conjunto de egresados de la Educación Básica Regular que tiene la intención de estudiar la carrera profesional, expresado como número de postulantes a la carrera profesional, lo cual puede aplicarse al conjunto de la oferta educativa o a la de una sola institución educativa.

El mercado ocupacional es la interacción entre la oferta ocupacional formada por los egresados de una carrera profesional y la demanda por estos egresados que requieran las empresas e instituciones. Si la oferta ocupacional es mayor que la demanda ocupacional, entonces habrá paro o desempleo entre los egresados de la carrera, caso contrario, entonces la oferta es deficitaria, faltando personal que ocupe los puestos demandados.

La relación entre los dos mercados señalados es que la demanda social por la carrera profesional después de los 5 o 6 años de su formación, se convierte en la oferta del mercado ocupacional. Ambos mercados y sus condiciones se respaldan mutuamente.

En el caso de la demanda social de la carrera de Contabilidad ofrecida por la Facultad de Ciencias
Contables de la UNMSM (Universidad del Perú, Decana de América), consideramos que sólo el 13\% de los postulantes a esta facultad alcanza vacante, comprobamos que la demanda social es la adecuada. Se comprueba que el ratio de demanda de admisión, que mide el nivel de selectividad de la carrera, es de 5.55, que determina que sólo 1 de cada 6 postulantes ingresa. Asimismo, en el diagnóstico realizado por la Facultad de Ciencias Contables referido al Plan Estratégico señala que, nuestros egresados tienen una rápida inserción en el mercado laboral. Sin embargo, nuestros instrumentos de recolección de información inquirirán sobre las condiciones determinantes de la demanda social y el mercado ocupacional, buscando determinar además, otras condiciones que pudieran faltar.

Proyecto Educativo - Currículo. Según el Modelo de Calidad de CONEAU, "el proyecto educativo se presenta en un documento denominado currículo de estudios. En él se encuentra la justificación de la carrera profesional, los perfiles del ingresante y del egresado, el plan de estudios y los contenidos de cursos o asignaturas".

También, señala que "Los principios y argumentos que justifican la carrera profesional son definidos sobre la base de la demanda social". 


\section{LA FORMACIÓN PROFESIONAL COMO PROCESO}

El Modelo de Calidad de CONEAU utiliza un enfoque de Proceso, como se observa en el Gráfico $\mathrm{N}^{\circ} 2$ que se presenta a continuación:

Es decir, Insumo - Proceso - Producto. En cuyo caso, considerando el proceso de enseñanza - aprendizaje de las carreras ¿Cuál es el insumo? y ¿Cuál es el producto? ¿Quién es nuestro cliente? ¿Nuestro producto es diseñado para satisfacer las necesidades del cliente?

\section{Gráfico $\mathrm{N}^{\circ} 2$ \\ PROCESO DE FORMACIÓN PROFESIONAL}

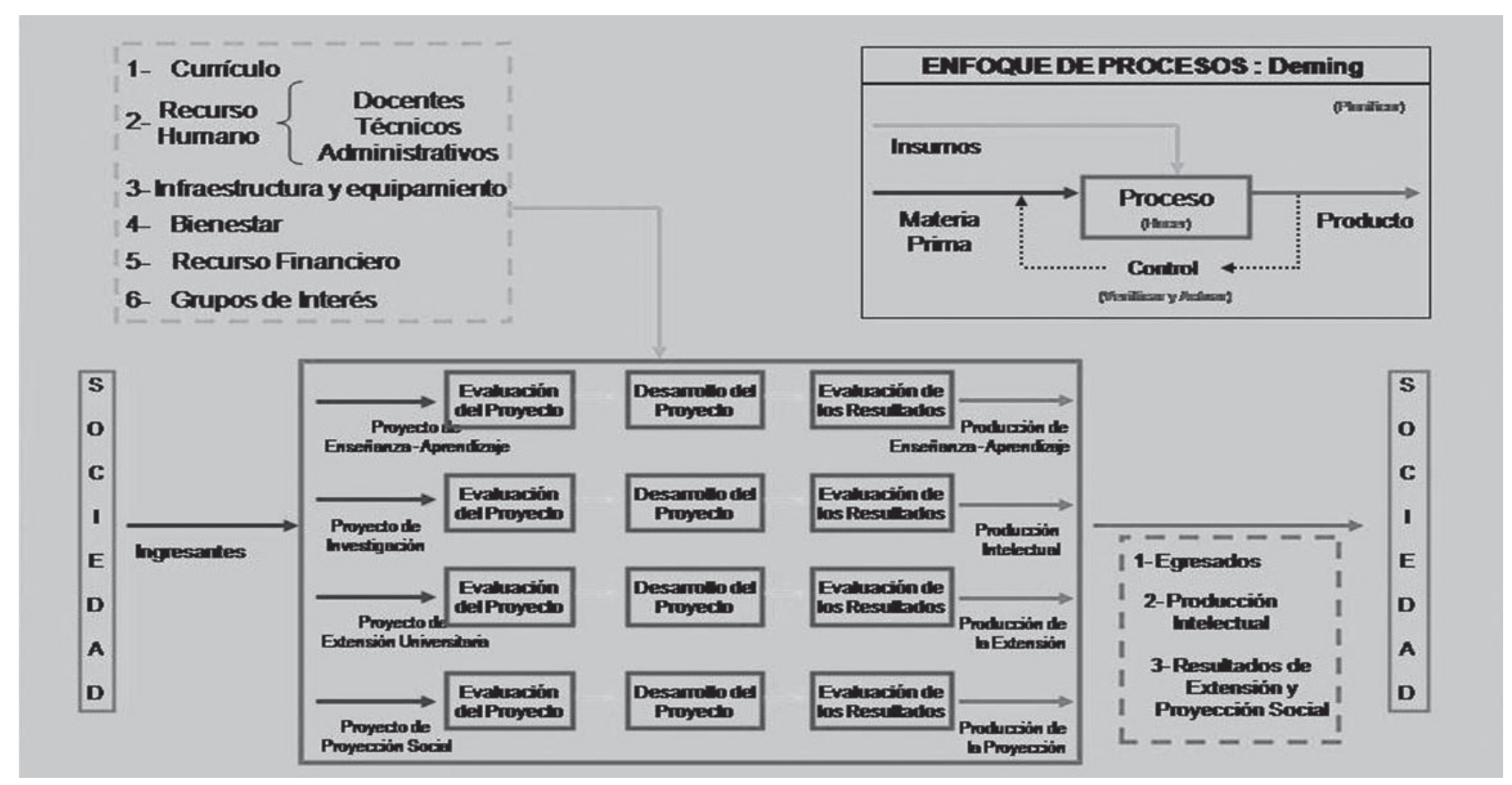

El insumo es la cualidad del ingresante (perfil del ingresante) el producto es el egresado con las competencias señaladas en el perfil del egresado, todos los detalles establecidos en el Plan Curricular. Como antecedente importante, citaremos a Flores, (2003) en la investigación titulada "Principales competencias profesionales requeridas al Contador Público Colegiado por las micro y pequeñas empresas del Perú", cuyas conclusiones deberán ampliarse y considerarse en la planificación curricular de la Facultad.

¿Cómo sabe la organización cuáles son las características del producto que debe producir? ¿Cómo sabe lo que desea el cliente?¿En qué momento pregunta al cliente?

En una empresa, las preguntas formuladas responde un estudio de mercado, el cual nos permite, entre otras cosas, conocer de nuestros clientes actuales potenciales y sus preferencias, lo que a su vez nos permite diseñar el producto para que cubran las expectativas.

Haciendo una analogía, una carrera profesional también debe formular un estudio de mercado. En nuestro caso, CONEAU propone un $\ll$ Estudio de la demanda social y mercado ocupacional de las carreras profesionales $\gg$.

\section{¿Quiénes son nuestros clientes?}

Los educandos. La proporción de postulantes sobre ingresantes es altísima, significando la aceptación de los clientes a nuestra oferta.

Los empleadores. Su aceptación se relacionará con el grado de empleabilidad de nuestros egresados y por los estudios de todo el mercado, como el de Pis- 
coya. Los empleadores representan grupos de interés, a quienes se les debe consultar para la planificación estratégica y curricular de la carrera.

La sociedad. Es el macro - cliente, con sus demandas de desarrollo y estabilidad. ¿Qué tipo de contador demanda las necesidades de desarrollo y estabilidad de la sociedad?

Otro aspecto crucial es el estudio de las condiciones futuras de la profesión, conforme a las tendencias económicas, sociales, tecnológicas, etc. Por ejemplo, ¿Cuáles son los cambios que impone a la profesión contable el rápido desarrollo de las tecnologías de la información y comunicación?.

\section{PRINCIPALES RESULTADOS}

Del planteamiento de los objetivos de la investigación, del marco teórico y conceptual presentados, y en base a su comparación con el diagnostico de la realidad de la carrera profesional ofrecida por nuestra Facultad, contenida en su Plan Estratégico, se ha identificado las variables de estudio, las dimensiones de las misma, y se ha procedido a la planificación del estudio de mercado.

Los investigadores hemos concluido en las siguientes determinantes de las variables de estudio, como sigue:
Determinantes de la demanda social de una carrera profesional.

1.Competencias logradas en la Educación Básica Regular.

2.Expectativas de empleo futuro (demanda ocupacional futura prevista).

3.Vocación personal.

4.Prestigio social de la carrera.

5.Duración y costo de la carrera profesional.

6.Oferta educativa.

\section{Determinantes del mercado ocupacional}

1.Número de graduados de la carrera profesional.

2. Tasa de crecimiento de los graduados de la carrera profesional.

3.Competencias requeridas a los profesionales empleados.

4.Nivel de ingreso de las empresas o instituciones demandantes.

5.Tasa de crecimiento económico general.

La presente investigación se propone resumir todos los aspectos obtenidos como conclusiones, además del Informe de Investigación en un «Plan de Trabajo para la formulación del estudio de demanda social y mercado ocupacional para la carrera profesional de Contabilidad», que está en estos momentos en proceso de elaboración.

El estudio de demanda social y mercado ocupacional tendrá las siguientes preguntas de investigación:

\section{Cuadro No 1}

\section{PROBLEMA DE INVESTIGACIÓN DE MERCADOS}

\section{PROBLEMA GENERAL}

¿Cuál es la demanda social y el mercado ocupacional de la carrera profesional de Contabilidad? PROBLEMAS ESPECÍFICOS

- ¿Cuáles son las características del servicio que presta la Facultad de Ciencias Contables?

- ¿Cómo es el desarrollo profesional de los egresados de la Facultad de Ciencias Contables en el periodo 2014 al 2024 ?

- ¿Cuál es la demanda por parte de los estudiantes de educación secundaria de Instituciones Educativas Privadas y Públicas para la formación profesional en Contabilidad?

- ¿Cuál es la demanda de las organizaciones por los profesionales de la carrera de Contabilidad?

- ¿Cómo es la oferta de la carrera de Contabilidad en Lima Metropolitana para el año 2014?

- ¿Cuál será la demanda futura de carrera de Contabilidad? 


\section{Cuadro No 2}

\section{CONTENIDO DEL ESTUDIO DE DEMANDA SOCIAL Y MERCADO OCUPACIONAL}

\section{1.- DEFINICIÓN DEL PROBLEMA DE INVESTIGACIÓN}
1. El problema de investigación.
2. Objetivos de la investigación.
3. Determinación de las variables - Operacionalización de las variables.

\section{2.- LA FACULTAD DE CIENCIAS CONTABLES Y LA CARRERA PROFESIONAL DE CONTABILIDAD}

1.La Facultad de Ciencias Contables de la UNMSM y la Escuela Académico Profesional de Contabilidad.

2.La Carrera Profesional de Contabilidad: Antecedentes y evolución.

3. Características de la formación profesional en Contabilidad de la Facultad de Ciencias Contables de la UNMSM.

4.Desarrollo profesional del egresado de la carrera profesional de Contabilidad de la Facultad de Ciencias Contables de la UNMSM.

\section{3.- EL MERCADO DE FORMACION PROFESIONAL - CARRERA PROFESIONAL DE CONTABILIDAD.}

1.Oferta educativa en la carrera profesional de Contabilidad en Lima Metropolitana.

2.Demanda de formación profesional - Carrera Profesional de Contabilidad.

3.Demanda específica de formación profesional - Carrera Profesional de Contabilidad - Facultad de Ciencias Contables - UNMSM.

4.Comparación Oferta - Demanda.

\section{4.- MERCADO OCUPACIONAL PARA LA CARRERA PROFESIONAL DE CONTABILIDAD.}

1.Determinantes de la demanda ocupacional de la Carrera Profesional de Contabilidad.

2.Demanda ocupacional histórica de la Carrera Profesional de Contabilidad en Lima Metropolitana.

3. Oferta Ocupacional histórica de la Carrera Profesional de Contabilidad en Lima Metropolitana.

4.Proyecciones de la oferta y demanda ocupacional de la Carrera Profesional de Contabilidad en Lima Metropolitana.

5.- ANÁLISIS DE LOS RESULTADOS.

6.- CONCLUSIONES Y RECOMENDACIONES.

\section{CONCLUSIONES}

1. Se aportan elementos conceptuales y metodológicos para la adecuada formulación de los estudios de la demanda social y del mercado ocupacional de las carreras de Contabilidad, lo que impactará positivamente en la planificación estratégica de la Facultad de Ciencias Contables de la Universidad Nacional Mayor de San Marcos. Específicamente, se han determinado las variables de estudio, las determinantes de estas variables, los objetivos del estudio, las preguntas de investigación y el contenido del estudio de demanda social y mercado ocupacional para la Carrera de Contabilidad.
Los elementos aportados preliminarmente por la investigación, se reunirán en un «Plan de Trabajo para la Formulación del Estudio de Demanda Social y mercado ocupacional para la carrera profesional de Contabilidad».

2. Dicho plan de trabajo deberá ser analizado por la Oficina de Calidad Académica y Acreditación de la Facultad de Ciencias Contables, para luego de su análisis y aportes, ser adoptado y elevado al Consejo de Facultad, para su realización oportuna.

3. Dichos hallazgos teóricos y metodológicos impactarán positivamente en la planificación curricular de la Facultad de Ciencias Contables de la Universidad Nacional Mayor de San Marcos. 
4. Dichos hallazgos teóricos y metodológicos y «Plan de Trabajo» impactarán positivamente en el proceso de autoevaluación y acreditación nacional de la Carrera Profesional de Contabilidad de la Facultad de Ciencias Contables de la Universidad Nacional Mayor de San Marcos.

\section{REFERENCIA BIBLIOGRÁFICA}

1. Estudio de la demanda Social para la Oferta de Carreras en la ESPOCH.(2008). Unidad Técnica de Planificación. Escuela Superior Politécnica de Chimborazo.

\section{REFERENCIAS HEMEROGRÁFICAS}

1. Guía de Acreditación de Carreras Profesionales Universitarias. (2009) CONEAU.

2. Estándares para la Acreditación de la Carrera Profesional de Contabilidad. CONEAU.

3. PISCOYA Luis. (2008) Formación universitaria vs. Mercado laboral II. Asamblea Nacional de Rectores. Perú.

4. Plan Estratégico 2012 - 2021. Facultad de Ciencias Contables - UNMSM.

5. Plan Estratégico Institucional 2012 -2021. Universidad Nacional Mayor de San Marcos. 